\title{
Causal Evidence for Mnemonic Metacognition in Human Precuneus
}

\author{
@Qun Ye (叶群), ${ }^{1 *}$ Futing Zou (邹富渟), ${ }^{1 *}$ Hakwan Lau (劉克頑), ${ }^{4,5,6,7} \mathrm{Yi} \mathrm{Hu}$ (胡谊), ${ }^{1}$ and $@$ Sze Chai Kwok (郭思齊) ${ }^{1,2,3}$ \\ ${ }^{1}$ Shanghai Key Laboratory of Brain Functional Genomics, Key Laboratory of Brain Functional Genomics Ministry of Education, School of Psychology and \\ Cognitive Science, East China Normal University, Shanghai 200062, China, ${ }^{2}$ Shanghai Key Laboratory of Magnetic Resonance, East China Normal \\ University, Shanghai 200062, China, ${ }^{3}$ NYU-ECNU Institute of Brain and Cognitive Science at NYU Shanghai, Shanghai 200062, China, ${ }^{4}$ Department of \\ Psychology, ${ }^{5}$ Brain Research Institute, University of California-Los Angeles, Los Angeles, California, 90095, ${ }^{6}$ Department of Psychology, University of Hong \\ Kong, Hong Kong, and 7 State Key Laboratory for Brain and Cognitive Sciences, University of Hong Kong, Hong Kong
}

Metacognition is the capacity to introspectively monitor and control one's own cognitive processes. Previous anatomical and functional neuroimaging findings implicated the important role of the precuneus in metacognition processing, especially during mnemonic tasks. However, the issue of whether this medial parietal cortex is a domain-specific region that supports mnemonic metacognition remains controversial. Here, we focally disrupted this parietal area with repetitive transcranial magnetic stimulation in healthy human participants of both sexes, seeking to ascertain its functional necessity for metacognition in memory versus perceptual decisions. Perturbing precuneal activity selectively impaired metacognitive efficiency of temporal-order memory judgment, but not perceptual discrimination. Moreover, the correlation in individuals' metacognitive efficiency between domains disappeared when the precuneus was perturbed. Together, these findings provide evidence reinforcing the notion that the precuneal region plays an important role in mediating metacognition of episodic memory retrieval.

Key words: confidence; episodic memory; metacognition; precuneus; TMS; visual perception

\section{Significance Statement}

Theories on the neural basis of metacognition have thus far been largely centered on the role of the prefrontal cortex. Here we refined the theoretical framework through characterizing a unique precuneal involvement in mnemonic metacognition with a noninvasive but inferentially powerful method: transcranial magnetic stimulation. By quantifying metacognitive efficiency across two distinct domains (memory vs perception) that are matched for stimulus characteristics, we reveal an instrumental role of the precuneus in mnemonic metacognition. This causal evidence corroborates ample clinical reports that parietal lobe lesions often produce inaccurate self-reports of confidence in memory recollection and establish the precuneus as a nexus for the introspective ability to evaluate the success of memory judgment in humans.

\section{Introduction}

Metacognition is the ability to introspectively monitor and control one's own cognitive processes, which is important to guide

\footnotetext{
Received March 10, 2018; revised June 6, 2018; accepted June 12, 2018.

Author contributions: Q.Y., F.Z., H.L., and S.C.K. edited the paper; F.Z. and S.C.K. wrote the first draft of the paper. Q.Y., F.Z., H.L., Y.H., and S.C.K. designed research; Q.Y. and F.Z. performed research; Q.Y. and F.Z. analyzed data; Q.Y., F.Z., H.L., and S.C.K. wrote the paper.

This work was supported by the National Institute of Neurological Disorders and Stroke of the National Institutes of Health Grant R01NS088628 (H.L.); by the Ministry of Education of PRC Humanities and Social Sciences Research Grant 16YJC190006, STCSM Shanghai Pujiang Program 16PJ1402800, STCSM Natural Science Foundation of Shanghai 16ZR1410200, NYU Shanghai, and the NYU-ECNU Institute of Brain and Cognitive Science at NYU Shanghai (S.C.K.). We thank Yudian Cai for programming the perceptual task, Xinming Xu for suggesting resolution comparison, and D. Jacob Gerlofs for proofreading.

The authors declare no competing financial interests.

${ }^{*}$ Q.Y. and F.Z. contributed equally to this work as co-first authors.

Correspondence should be addressed to Dr. Sze Chai Kwok, East China Normal University, Shanghai 200062, China. E-mail: sze-chai.kwok@st-hughs.oxon.org.
}

adaptive behavior, social interaction and mental health (Flavell, 1979; Nelson, 1990; Teasdale et al., 2002; Frith, 2012). Metacognitive capacity has been mostly assessed by self-reporting of level of confidence in one's own decisions that correlate with objective performance. The initial task is often called a "type 1 task" and the ensuing confidence judgment task is called a "type 2 task" (Galvin et al., 2003). A widely used approach to estimate metacognitive efficiency without having it confounded by the primary task performance and response bias is to calculate the comparison between type 1 sensitivity $\left(d^{\prime}\right)$ and type 2 sensitivity (meta- $d^{\prime}$ ). This approach can quantify meta-ability under signal detection theory (SDT) framework (Maniscalco and Lau, 2012) or by a recently 
developed hierarchical Bayesian estimation method (Fleming, 2017).

Despite a large amount of recent research showing the neural architecture of metacognition in various cognitive domains, like visual perception and memory (Fleming et al., 2010, 2014; Yokoyama et al., 2010; Baird et al., 2013; McCurdy et al., 2013; Rahnev et al., 2016), the underlying mechanisms of metacognition are incompletely understood. A central question is whether human metacognition depends on some domain-general neural structures, or is it supported by domain-specific components? Although it has been reported that metacognitive behavioral indices are correlated across memory versus perception domains (McCurdy et al., 2013), their functional neural correlates might be largely independent (Baird et al., 2013).

In contrast to the established role of the anterior prefrontal cortex in perceptual metacognition (Fleming et al., 2014), compelling evidence has converged to reveal an important role of the precuneus in memory metacognition (Fleck et al., 2006; Fleming et al., 2010; McCurdy et al., 2013). Functionally, it has been shown that task-related activity in the precuneus was greater during memory tasks compared with during perceptual decisions (Morales et al., 2018). Anatomically, structural variation in the precuneal region was correlated more robustly with memory metacognitive efficiency than with visual perceptual metacognitive efficiency, ascribing a critical role of the precuneus in metamemory (McCurdy et al., 2013).

The extant evidence for the function of the precuneus in metacognition has been correlational. Here we used a disruptive technique that can noninvasively establish the causal role of the precuneus in metacognition across the memory and perceptual domains. We applied transcranial magnetic stimulation (TMS) over either the precuneus or a control site before the type 1 tasks to perturb the neural activity so as to ascertain whether the precuneus might be causally involved in metacognition in either or both domains. In both tasks, on each trial the participants were required to make a two-alternative forced-choice judgment between a pair of still frames, followed by a confidence rating of their choice decision for that trial; the only difference between the two tasks was the task demands. In the memory task, the participants were asked to identify the image that was presented earlier in a video gameplay they had encoded $24 \mathrm{~h}$ before; in the visual perceptual task, the same group of participants were required to discriminate the difference in resolution between the two images. We kept the individual sets of pair-images identical in both tasks per participant. To anticipate, we expected a Task $\times$ TMS interaction, which shall arise from a more pronounced deficit in metamemory efficiency following TMS to the precuneus than in metaperceptual efficiency.

\section{Materials and Methods}

\section{Participants}

18 adults ( 7 female, age 19-24 years) from the student community of the East China Normal University participated in this study. Each of them participated in both tasks, giving us a within-subjects comparison. All participants had normal or corrected-to-normal vision, no reported history of neurological disease, no other contraindications for MRI or TMS, and all gave written informed consent. They were compensated financially for their participation. No subject withdrew due to complications from the TMS procedures, and no negative treatment responses were observed. The study was approved by University Committee on Human Research Protection of East China Normal University.

\section{Overview of study}

The memory task and perceptual task were each separated into two experimental sessions. Immediately before performing the main task, the participants received $20 \mathrm{~min}$ of repetitive TMS that targeted at one of the two cortical sites (within-subjects: TMS-vertex vs TMS-precuneus) in a counterbalanced manner (Fig. 1A, Session 1 and Session 2). The session order; numbers of trials; dimension, position and sequence of stimulus presented; response time allowed for the type 1 task judgment; and intertrial intervals were all identical in both tasks. Highresolution structural scans were acquired for each participant to guide the TMS procedure.

\section{Experimental design and statistical analysis}

Tasks and procedure. Each participant completed 480 trials in total in each of the two tasks, both of which lasted $\sim 45 \min (2$ sessions $\times 4$ blocks $\times 60$ trials per block).

The memory task required participants to choose the image that happened earlier [temporal order judgment (TOJ)] in the video game they had played $1 \mathrm{~d}$ before. The visual stimuli were presented using E-prime software (Psychology Software Tools), as back-projected via a mirror system to the participant. Each trial was presented for $5 \mathrm{~s}$ during which participants performed the TOJ. They were then allowed $3 \mathrm{~s}$ to report their confidence level following the memory judgment. Participants performed the TOJ task using their index and middle fingers of one of their hands via an MRI compatible five-button response keyboard (Sinorad). The participants reported their confidence level ("Very Low", "Low", "High", or "Very High") regarding their own judgment of the correctness of TOJ with four fingers of the other hand. The left/right hand response contingency was counterbalanced across participants. The participants were encouraged to report their confidence level in a relative way and make use of the whole confidence scale. Following these judgments, a fixation cross with a variable duration (1-6 s) was presented (Fig. 1C). Regarding the memory task, we have also set out to examine the multivoxel pattern representation of the temporal distances between the two frames during the TOJ memory task and thus we administered the memory task inside an MRI scanner (fMRI results reported previously). By contrast, because of cost consideration for neuroimaging here we focused on an issue that has interested us scientifically, that is whether TMS on the precuneus would produce differential effects on memory versus perception meta-ability, and thus we did not scan the participants again during the perceptual task.

The same sets of paired images were used in the perceptual task, in which the participants were required to choose either the clearer (or blurrier, counterbalanced across participants) image among a pair of images on each trial. The participants made an image-resolution comparison judgment and then a confidence rating of their type 1 task decision (Fig. 1D) with a 17 inch CRT monitor in a dimly illuminated room. There was a practice block before each session for the participant to get familiar with the task demands.

Quantification of metacognitive efficiency. Memory and perceptual performance were quantified using the percentage of correct judgments and the $d^{\prime}$ of type 1 signal detection theory (Green and Swets, 1966). We evaluated the metacognitive ability of both tasks by meta- $d^{\prime}$. Meta- $d^{\prime}$ quantifies metacognitive sensitivity (the ability to discriminate between correct and incorrect judgments) in a SDT framework. Meta- $d^{\prime}$ was widely used as a measure of metacognitive capacity because it is expressed in the same scale as $d^{\prime}$, so the type 2 sensitivity (meta- $d^{\prime}$ ) could be compared with the type 1 sensitivity $\left(\mathrm{d} d^{\prime}\right)$ directly (Maniscalco and Lau, 2012; Fleming and Lau, 2014). If meta- $d^{\prime}$ is equal to $d^{\prime}$, it means that the metacognitive sensitivity is ideal. Here, we calculated the meta- $d^{\prime}-d^{\prime}$, a metric for estimating the metacognitive efficiency (level of metacognition given a particular level of performance or signal processing capacity (Rounis et al., 2010). Moreover, we computed metacognitive efficiency using a hierarchical Bayesian estimation method, which can avoid edgecorrection confounds and enhance statistical power (Fleming and Daw, 2017). Following a common practice in the literature (Fleming et al., 2014; Faivre et al., 2018), we performed the analyses using both 4-point confidence ratings and a synthetic two-category (high and low) ratings. Notably, responders do use the 4-point scale rather differently (e.g., some people may only use 1,2,3 more, whereas others use $2,3,4$ ). The practice of down-sampling to 2-point makes use of the median split, which helps to reduce bias regarding how the subjects use the scale. 
A

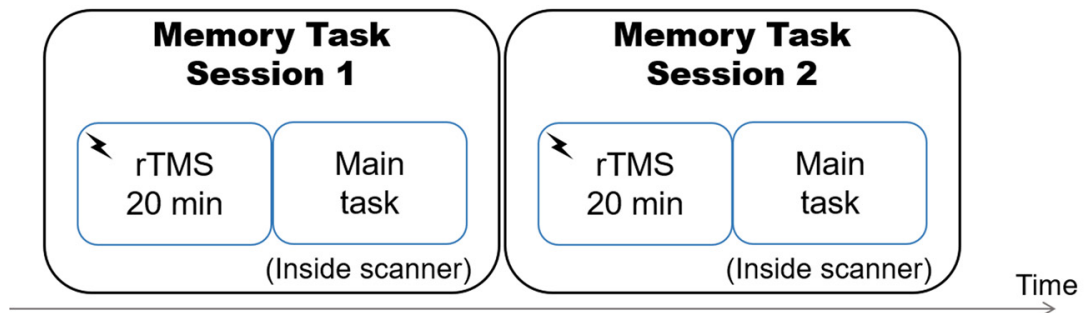

B

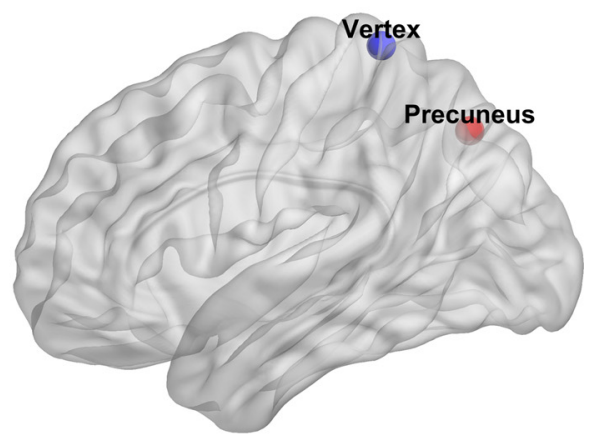

C

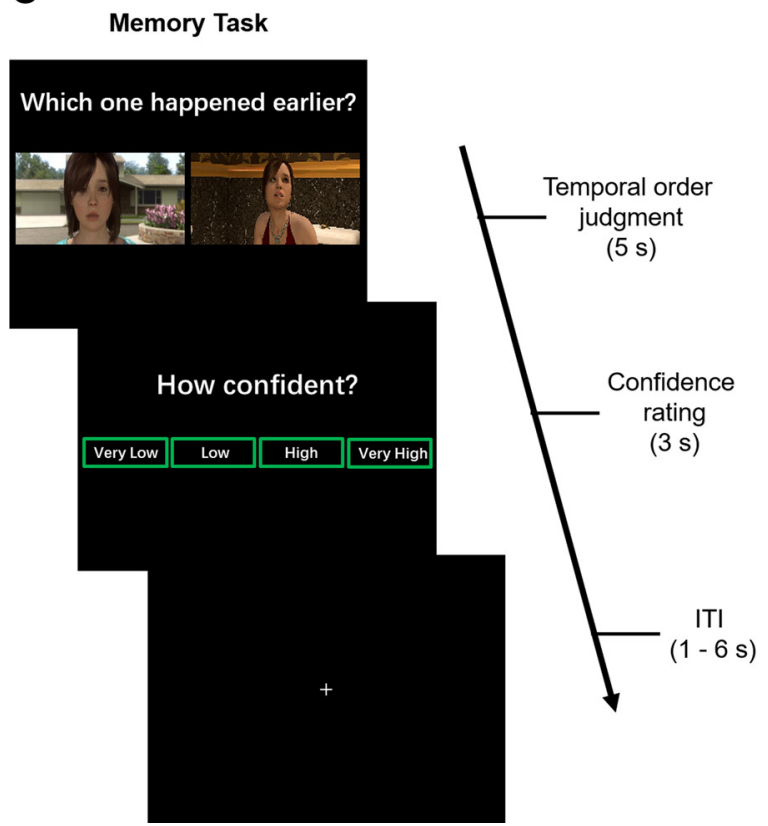

D

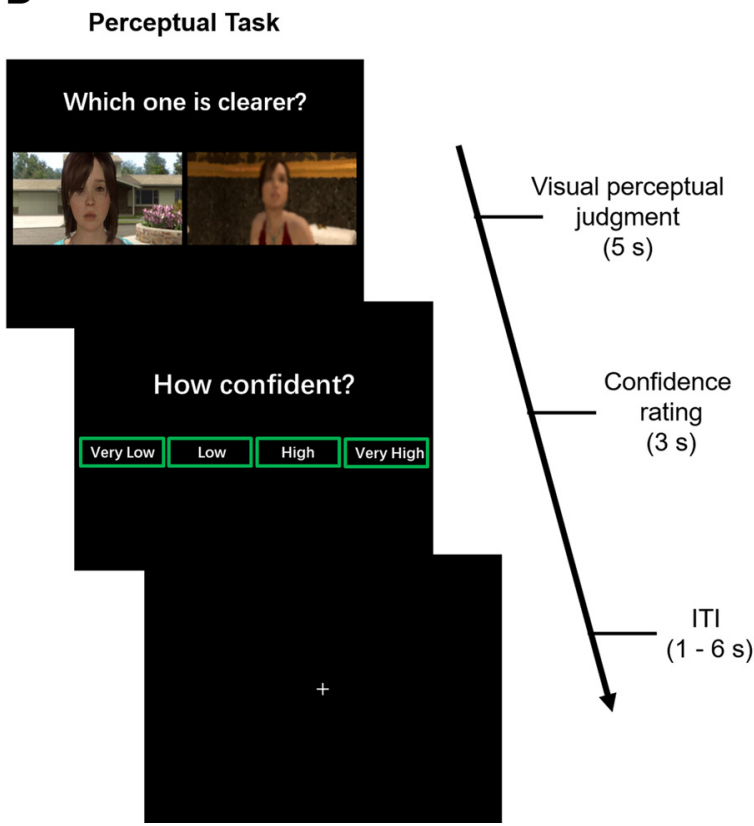

Figure 1. Study design. $A$, Experimental overview. In experimental Sessions 1 and 2 of each task, participants received 20 min of rTMS to either one of two cortical sites before performing the main task. The stimulation sites (within-subjects design: TMS-precuneus vs TMS-vertex) and choices of video game chapters were counterbalanced within subjects across task. $\boldsymbol{B}$, Location of precuneus (target site) is depicted in red and vertex (control site) in blue. The target site for precuneus stimulation (MNI $x, y, z=6,-70,44)$ was based on Kwok et al. (2012). C, In the memory task, the participants performed a temporal order judgment task, by choosing the image that happened earlier in the video game. $\boldsymbol{D}$, In the perceptual task, participants identified which frame of the two was clearer (or blurrier). After the type 1 tasks, participants rated their confidence level on a 4-point scale. ITI, intertrial interval.

Both meta- $d^{\prime}$ and $d^{\prime}$ measures assume that the variance of the internal response takes a Gaussian distribution, and importantly, that the distributions associated with the two type 1 responses respectively are of equal variance. To ensure our results were not due to any idiosyncratic violation of the assumptions of SDT, we additionally calculated the $\phi$ coefficient index, which does not make these strong assumptions (Fleming and Lau, 2014). Rather, it evaluates how optimally each trial was assigned for high or low confidence based on performance in the preceding cognitive judgment, reflecting the association between the two binary variables (Kornell et al., 2007). The coefficient was calculated by the following equation using the number of trials classified in each case $[n($ case $)]$ :

phi coefficient $(\Phi)=\frac{n(\text { Correct High }) \times n(\text { Incorrect Low })-n(\text { CorrectLow }) \times n(\text { Incorrect High })}{\sqrt{n(\text { Correct }) \times n(\text { Incorrect }) \times n(\text { High }) \times n(\text { Low })}}$

Data were processed with in-house software on MATLAB and statistical inference was made using RStudio. Trials missing either one of the mea- sures (memory: $2.9 \%$ of TOJ trials, $2.2 \%$ confidence rating; perception: $0.7 \%$ in type 1 trials) were excluded from the analysis.

\section{Stimuli}

Stimuli were extracted from an action-adventure video game (Beyond: Two Souls), which was created by the French game developer Quantic Dream and played in the PlayStation 4 video game console developed by Sony Computer Entertainment. Participants played 14 chapters in total across two sessions: 7 in experimental Session 1 and then another 7 in Session 2. These subject-specific video were recorded and were used for extraction of still images for the tasks.

For the memory task, we selected static images from the subjectspecific recorded videos which the participants had played the day before. Each second in the video consisted of 29.97 static images (frames). In each game-playing session, 240 pairs of images were extracted from the seven chapters and were paired up for the task based on the following 
criteria: (1) the two images had to be extracted from either the same chapters or adjacent chapters (Within- vs Across-chapter condition); (2) the temporal distance (TD) between the two images were matched between Within- and Across-chapter conditions; and (3) to maximize the range of TD, we first selected the second longest chapter of the video and determined the longest TD according to a power function ( power $=1.5$ ), at the same time ensuring the shortest TD to be longer than 30 frames. We generated 60 progressive levels of TD among these pairs.

For the perceptual task, the same sets of subject-specific stimuli from the memory task were used. On each trial, the resolution of one of the images was reduced using Python Imaging Library through resizing the image to change the pixel dimension. For instance, setting an image to three-tenths of the original size changed the pixel dimension to threetenths, then the image was resized to its primary size so that the pixels per inch (PPI) decreased proportionately. The higher the PPI, the smaller the difference between the image resolution of the resized one and the original was, which also meant this pair would be harder to discriminate than another pair with a lower PPI value. Based on participants performance in the memory task, we predetermined five difficulty levels for the perceptual task ( $n=1 \sim 5 ; 1$ being the hardest). The image resolution was adjusted online using an $n$-down/1-up adaptive staircase procedure, aiming to equate individual performance with his or her performance in the memory task.

\section{Anatomical MRI images}

A 3-tesla Siemens Trio magnetic resonance imaging scanner (Siemens Medical Solutions) was used to acquire high-resolution T1-weighted images for each participant (192 sagittal slices, TR $=2530 \mathrm{~ms}$, TE $=2.34$ $\mathrm{ms}$, TI $=1100 \mathrm{~ms}$, flip angle $=7^{\circ}, \mathrm{FOV}=256 \times 256 \mathrm{~mm}, 0.9 \mathrm{~mm}$ thickness, voxel size $=1 \times 1 \times 1 \mathrm{~mm}$ ) to stereotaxically guide the transcranial stimulation.

\section{Repetitive TMS: procedure, protocol, and sites}

TMS is a method of noninvasive cortical stimulation that can modulate cognitive functions. Previous studies have demonstrated that repetitive stimulation with TMS over the precuneus (Kraft et al., 2015) or lateral parietal cortices (Wang et al., 2014; Nilakantan et al., 2017) produce robust effects on memory related ability, showing the efficacy of repetitive TMS (rTMS) targeted at relatively deep regions.

rTMS was applied using a Magstim Rapid ${ }^{2}$ magnetic stimulator connected to a $70 \mathrm{~mm}$ double air film coil. The structural T1-weighted magnetic resonance images obtained from each subject were used in Brainsight2.0, a computerized frameless stereotaxic system (Rogue Research), to localize the target brain regions. Target stimulation regions for rTMS were selected in the system by transformation of the Montreal Neurological Institute (MNI) stereotaxic coordinates to participant's normalized brain. The sites stimulated were located in the precuneus at the MNI coordinate $x=6, y=-70, z=44$ (Kwok et al., 2012), and in a control area on the vertex, which was identified at the point of the same distance to the left and the right pre-auricular, and of the same distance to the nasion and the inion (Fig. 1B). For combining each subject's head with the MRI images, location information of each subject's head was obtained individually by touching four fiducial points, which are the tip of the nose, the nasion, and the intertragal notch of each ear using an infrared pointer. The real-time locations of reflective markers which were attached to the coil and the subject were monitored by an infrared camera using a Polaris Optical Tracking System (Northern Digital).

In each session, TMS was delivered to either the precuneus or vertex before the main task. TMS was applied at $1 \mathrm{~Hz}$ frequency for a continuous duration of $20 \mathrm{~min}$ (1200 pulses in total) at $110 \%$ of active motor threshold (MT), which was defined as the lowest TMS intensity delivered over the motor cortex necessary to elicit visible twitches of the right index finger in at least 5 of 10 consecutive pulses (Rossini et al., 2015). The MT was measured at the beginning of experiment Session 1 in both the memory and perceptual tasks. The order of stimulation sites was counterbalanced within subjects across tasks. During stimulation, participants wore earplugs to attenuate the sound of the stimulating coil discharge. The coil was held to the scalp of the participant with a custom coil holder and the subject's head was propped a comfortable position. Coil orientation was parallel to the midline with the handle pointing downward. Immediately after the 20 min of rTMS, subjects performed four blocks of memory task in the MRI scanner (mean delay from rTMS to beginning of test: TMSprecuneus $=15.29 \mathrm{~min}$, TMS-vertex $=20.76 \mathrm{~min}$ ), or performed a visual perceptual task in a psychophysics room (mean delay from rTMS to beginning of test: TMS-precuneus $=6.7 \mathrm{~min}$, TMS-vertex $=6.3 \mathrm{~min}$ ). This particular stimulation magnitude and protocols of rTMS (lowfrequency stimulation of $1 \mathrm{~Hz}$ ) is known to induce efficacious intracortical inhibitory effects for over 60 min (Iyer et al., 2003; Rossi et al., 2009; Thut and Pascual-Leone, 2010). Given that both of our tasks lasted 45 min, the TMS effects should have been long-lasting enough for the tasks. Although these inhibitory effects are also known to level off within hours by the end of the stimulation, for safety reason and to avoid carryover effects of rTMS across sessions, experimental Sessions 1 and 2 were conducted on 2 separate days for both tasks (memory: mean interval $=8 \mathrm{~d}$; perceptual: mean interval $=3.9 \mathrm{~d}$ ).

\section{Results}

We aimed to verify the causal role of the precuneus in subserving the metacognition of memory and perception processes. The following results cover the effects of TMS on type 1 and type 2 task performances. The mean percentages correct were $67.41 \%$ (SD = $7.22 \%)$ and $75.77 \%(\mathrm{SD}=4.75 \%)$ for the memory and perception tasks, respectively. We then examined whether the type 1 task performance and mean confidence ratings might be affected by TMS. As expected, task performance and mean confidence ratings were not different between the two TMS conditions in neither memory (paired $t$ test, accuracy, $t_{(17)}=0.349, p=0.640$; $\mathrm{RT}, t_{(17)}=1.997, p=0.090$; confidence rating, $t_{(17)}=0.069, p=$ 0.780 ) nor perceptual part (paired $t$ test, accuracy, $t_{(17)}=1.091$, $p=0.480 ; \mathrm{RT}, t_{(17)}=0.842, p=0.490$; confidence rating, $t_{(17)}=$ $0.461, p=0.560$; Fig. $2 A-C)$. Despite the differences in first-order task performance, we see that these first-order performances were not diminished or changed substantively by TMS (precuneus vs vertex) in either condition. This lack of effect is not trivial, because even though the first-order performances were not exactly the same, they were near threshold (71\%) in both cases, meaning there should have been some sensitivity in detecting an effect should TMS be detrimental to first-order performance in either the memory or perception conditions. We also reported the level of difficulty provided in each condition. We quantified the memory task difficulty by temporal distances between the pairs of images, and the perceptual task difficulty using the difference in resolution (ratio) between the pairs of images. There were no significant differences in the mean level of task difficulty provided between the two TMS sites in either the memory task (paired $t$ test, $t_{(17)}=-0.798, p=0.436$; Fig. $2 D$ ) or the perceptual task (paired $t$ test, $t_{(17)}=-1.305, p=0.209$; Fig. $2 E$ ).

We then used a robust metacognitive index (meta- $\left.d^{\prime}-d^{\prime}\right)$ to investigate whether TMS on the precuneus might affect metacognitive performance on the tasks. We performed a 2 (Task: memory/perception) $\times 2$ (TMS: precuneus/vertex) repeatedmeasures ANOVA for metacognitive efficiency, quantified as meta- $d^{\prime}-d^{\prime}$, from the SDT-based model and the hierarchical model separately. In the SDT-based model, we found an interaction effect between Task and TMS site $\left(F_{(1,17)}=7.25, p=0.015\right.$; Fig. $3 A$ ). The interaction was driven by lower metacognitive efficiency following TMS to precuneus relative to TMS to vertex in the memory task (paired $t$ test, $t_{(17)}=-2.155, p=0.046$ ), whereas no difference in metacognitive efficiency was found in the perceptual task (paired $t$ test, $t_{(17)}=1.378, p=0.186$ ). Metacognitive efficiency using the hierarchical model revealed the same pattern of results (Task $\times$ TMS interaction: $F_{(1,17)}=7.312$, $p=0.015$; memory: paired $t$ test, $t_{(17)}=-2.119, p=0.049$; 
A

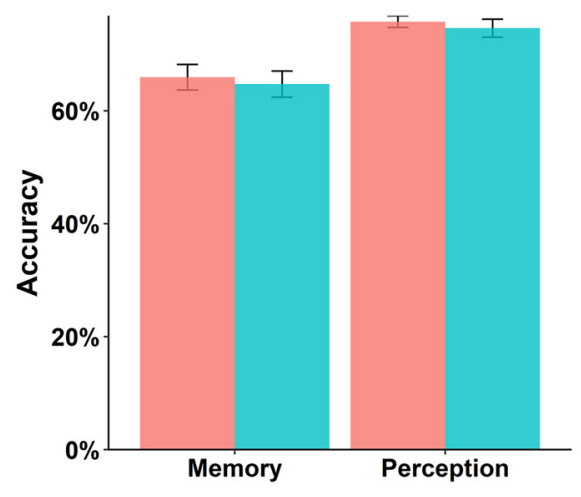

D

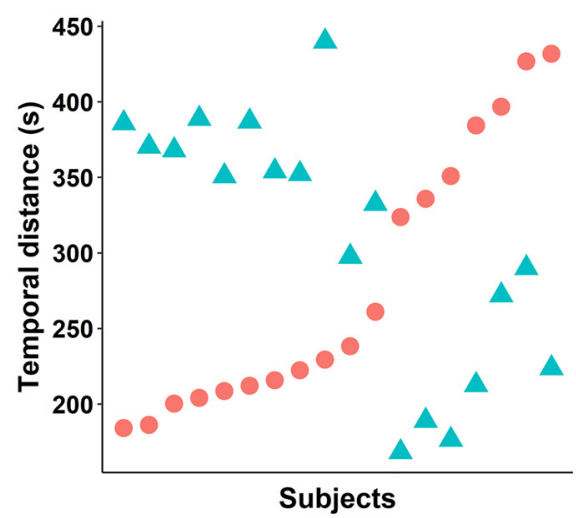

B

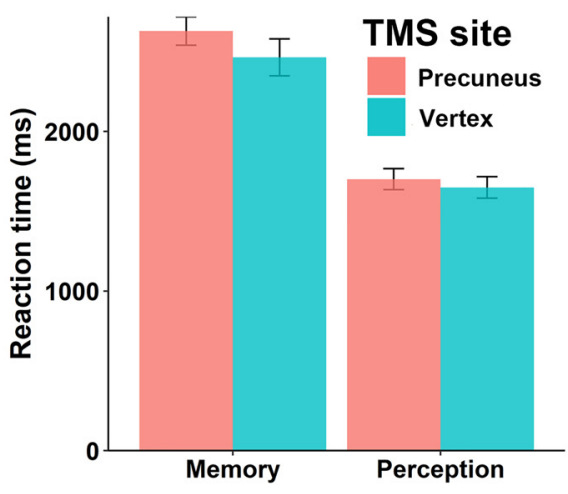

C

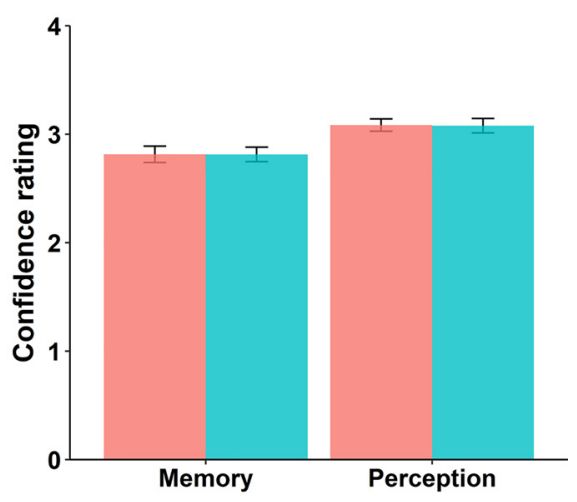

$E$
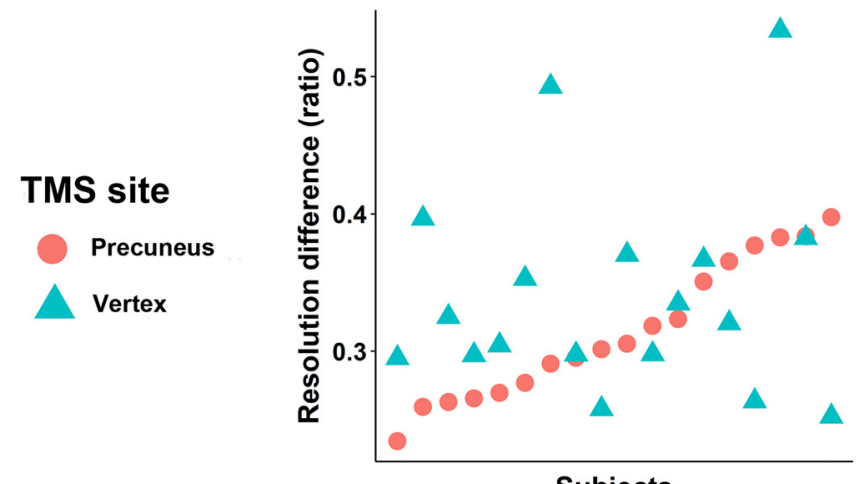

Figure 2. Basic task performance and task difficulty. Type 1 task performance and mean confidence ratings were not affected by TMS in either of the tasks: $(\boldsymbol{A})$ accuracy, $(\boldsymbol{B})$ reaction time, and $(\boldsymbol{C})$ mean level of confidence ratings. $\boldsymbol{D}$, Memory task difficulty provided for each participant: mean level of temporal distance between pair of images. $\boldsymbol{E}$, Perceptual task difficulty provided for each participant: mean level of difference in resolution (ratio) between pair of images. Error bars denote the SEM over participants.

perception: paired $t$ test, $t_{(17)}=1.334, p=0.200$; Figure $\left.3 B\right)$. We also performed the same sets of analyses using 4 confidence levels. This set of results similarly revealed a Task $\times$ TMS interaction with the SDT-based model $\left(F_{(1,17)}=4.506, p=0.049\right)$ and with the hierarchical model $\left(F_{(1,17)}=4.051, p=0.060\right)$. The results are essentially identical with using four confidence levels versus two confidence levels. In addition, the same significant Task $\times$ TMS interaction was also replicated with another widely used index, $\log \left(\right.$ meta- $\left.d^{\prime} / d^{\prime}\right)$ with the SDT-based model $\left(F_{(1,17)}=5.330, p=\right.$ 0.034; Fig. $3 C)$ and with the hierarchical model $\left(F_{(1,17)}=5.798\right.$, $p=0.028$; Fig. 3D).

To add credibility to these results, we replicated these findings with the Phi coefficient (ANOVA, $F_{(1,17)}=13.81, p=0.002$; Fig. $3 E$ ), confirming that our results were not biased by any idiosyncratic violations of the assumptions of SDT. These findings of lower metacognitive efficiency in the memory task following TMS to the precuneus compared with the vertex confirm our prediction that the precuneus causally mediates memory metacognition, but not perceptual metacognition. To better characterize the effect of TMS on metacognitive efficiency, we performed sign tests to verify the extent of changes between TMS to precuneus and vertex. Metacognitive efficiency was reduced by TMS to the precuneus in the majority of participants in the memory task ( $13 / 18$ reduced, $p=0.035$, sign test), but not in the perceptual task (10/18 reduced, $p=0.290$, sign test).
These meta-indices are in principle based on how people rate their confidence, which refer to how meaningful a person's confidence rating is in distinguishing between correct and incorrect responses. We accordingly ran a three-way repeated-measures ANOVA (Task: Memory/Perception $\times$ TMS: precuneus/vertex $\times$ Confidence: Low/High) on the type 1 task percentage correct and obtained a significant three-way interaction $\left(F_{(1,17)}=\right.$ $10.652, p=0.005)$. The TMS effect was disproportionally stronger in the memory task, as evident in a TMS $\times$ Confidence interaction $\left(F_{(1,17)}=4.487, p=0.049\right.$; Fig. $\left.3 F\right)$, than in the perceptual $\operatorname{task}\left(F_{(1,17)}=1.24, p=0.281\right.$; Fig. $\left.3 G\right)$. Such effects in the memory task were driven by higher accuracy following TMSprecuneus than TMS-vertex in the low confidence rating condition (paired $t$ test, $t_{(17)}=2.354, p=0.031$ ), but not in the high confidence rating condition (paired $t$ test, $t_{(17)}=-0.4, p=$ 0.694 ). This finding indicates that TMS targeted at the precuneus affects the efficacy of confidence ratings such that low confidence responses are more accurate on type 1 memory task following TMS to the precuneus versus the vertex, and this is not seen in the perceptual task or for high confidence trials.

To further probe whether the TMS effect on memory metacognition would be reflected by within-subjects changes in the between-tasks covariations, we calculated the between-tasks (Memory/Perception) correlations for all individuals' type 1 task sensitivity $\left(d^{\prime}\right)$ and metacognitive efficiency respectively. We 
A

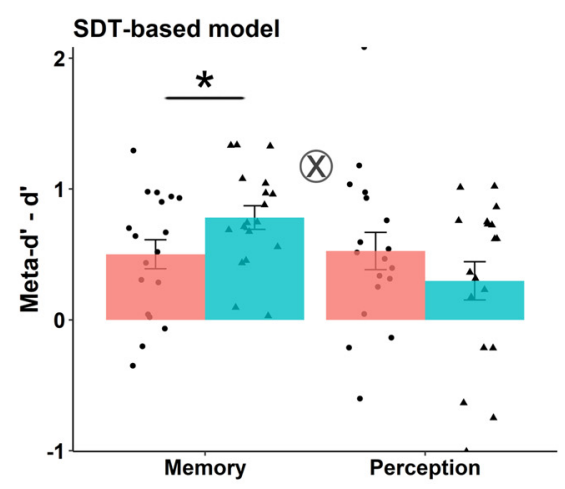

C

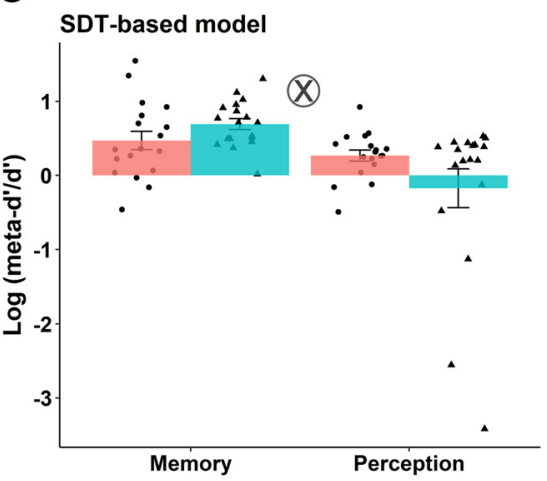

F

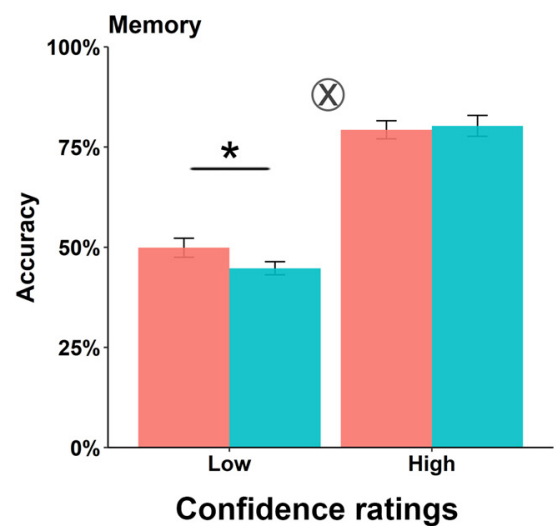

B

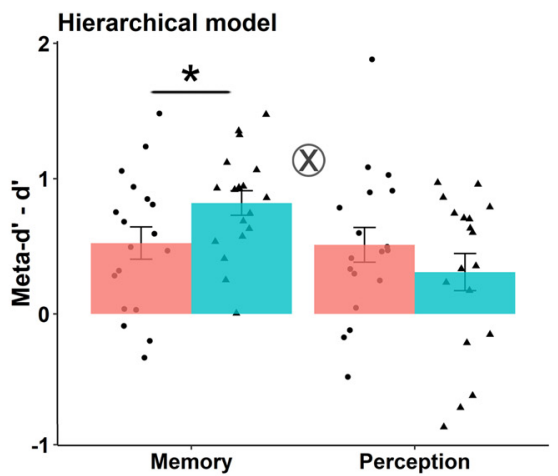

D

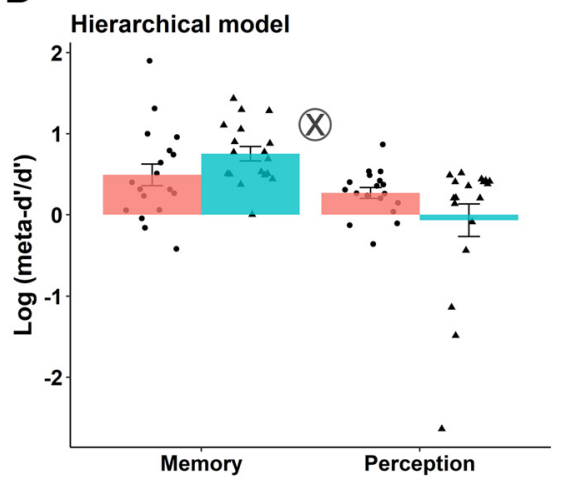

E

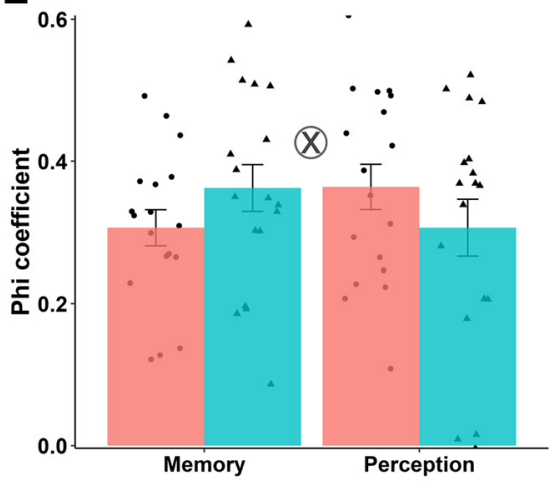

TMS site

Precuneus

Vertex
G

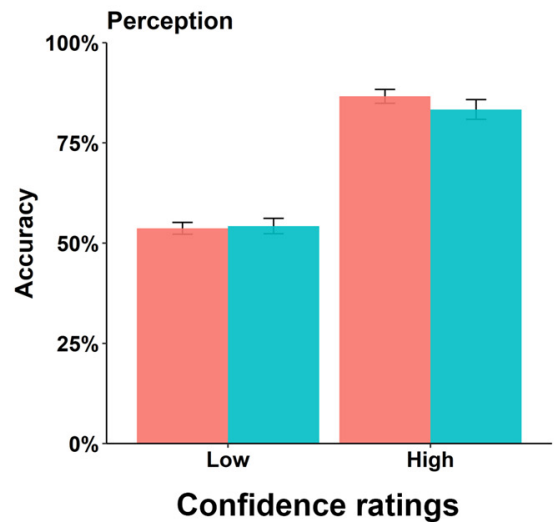

Figure 3. Differential effects of TMS on metacognitive performance. $\boldsymbol{A}$, Metacognitive efficiency in SDT-based model (meta- $d^{\prime}-d^{\prime}$ ) under TMS-precuneus was lower than metacognitive efficiency under TMS-vertex in memory task but not in perceptual task. $\boldsymbol{B}$, Metacognitive efficiency in hierarchical model under TMS-precuneus was lower than metacognitive efficiency under TMS-vertex in memory task but not in perceptual task. C, TMS $\times$ Task interaction in logarithm of meta- $d^{\prime} / d^{\prime}$ computed with the SDT model. $\boldsymbol{D}$, TMS $\times$ Task interaction in logarithm of meta- $d^{\prime}$ / $d^{\prime}$ computed with the hierarchical model. $\boldsymbol{E}$, TMS $\times$ Task interaction in $\phi$ coefficient (the correlation between accuracy and confidence over trials). $\boldsymbol{F}$, TMS $\times$ Confidence ratings interaction in memory task. The type 1 accuracy for low confidence ratings under TMS-precuneus is significantly higher than that under TMS-vertex; no such effect for high confidence ratings. $\mathbf{G}$, No significant effect of TMS in perceptual task. $\otimes$ indicates significant interaction $p<0.05,{ }^{*} p<0.05$. Error bars represent SEM over participants.

found that participants' type 1 sensitivity $\left(d^{\prime}\right)$ between the perceptual and memory tasks are positively correlated, and that the magnitude of the correlation was not affected by TMS (TMSvertex: $r=0.90, p<0.001$; TMS-precuneus: $r=0.82, p<0.001$; comparison between correlations: $z=-0.86, p=0.390$; Fig. $4 A$ ). This again indicates that TMS had no effect on the basic task performance, in line with the pattern shown in Figure 2. In contrast, whereas the metacognitive efficiency for the two tasks were significantly correlated in the control (TMS-vertex) condition, much like what was reported previously (McCurdy et al., 2013), such correlational pattern was notably eliminated under TMSprecuneus treatment, and the correlation coefficient was significantly lower than that of the TMS-vertex condition (TMS-vertex: $r=0.72, p<0.001$; TMS-precuneus: $r=-0.13, p=0.63$; comparison between correlations: $z=-3.38, p<0.001$; Fig. $4 B$ ). Together, these results reveal that TMS to the precuneus affects the metacognitive performance specifically for the memory domain. 
A

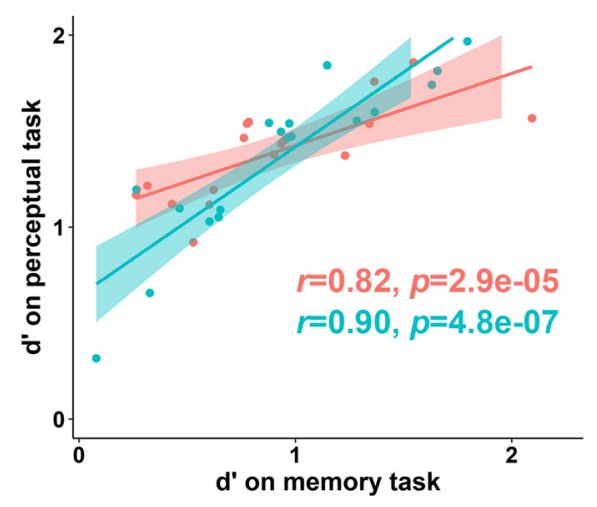

B

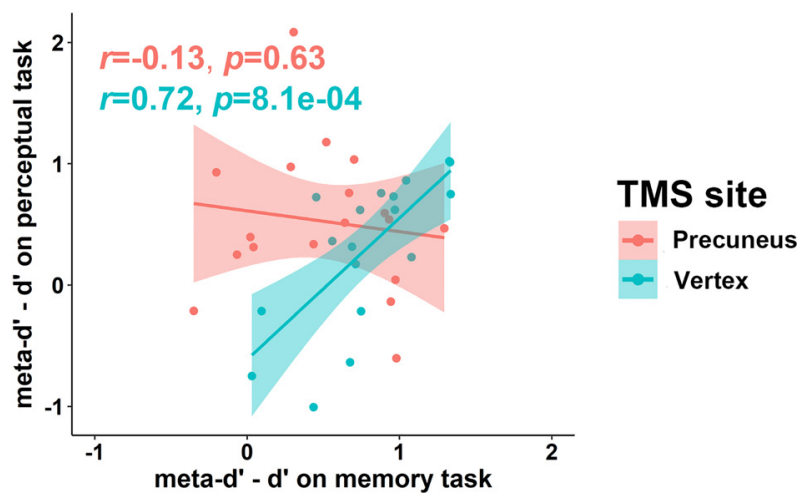

Figure 4. Correlation between memory and perceptual task performance and metacognitive indices. $\boldsymbol{A}$, TMS had no effect on the participants' type 1 sensitivity ( $d^{\prime}$ ). The positive correlation between $d^{\prime}$ on the perceptual and memory tasks was not affected by TMS. $\boldsymbol{B}$, In the TMS-vertex condition, the metacognitive efficiencies across the group were significantly correlated between memory and perceptual tasks. However, following TMS-precuneus, such between-tasks metacognitive efficiencies were no longer correlated.

\section{Discussion}

We used an inferentially powerful technique to investigate the critical role of the precuneus in metacognitive ability in two distinct domains: memory and perception. We demonstrated that magnetic field stimulation targeted at the precuneus impairs metacognitive efficiency in a long-term memory task without eliciting amnesia. The TMS's task-specific effect on the memory task implies that part of the neurobiological prerequisite for metamemory ability, especially those in relation to episodic recollection/TOJ, might be housed in the precuneus.

The precuneus is known to be implicated in memory metacognition, as suggested by correlative evidence coming from anatomical connectivity and related functional activity analyses. For instance, a previous study identified a link between memory metacognitive efficiency and the precuneal gray matter density in healthy individuals (McCurdy et al., 2013), whereas a similar relationship was found between mnemonic metacognitive efficiency and resting-state functional connectivity between the precuneus and medial aPFC (Baird et al., 2013). Considering that no prior study has executed controlled, targeted perturbation on this medial parietal region, we thus set out to examine its functional necessity for mnemonic metacognition by disrupting the precuneal function with TMS.

Here, consistently across several metacognition metrics, our TMS-induced focal disruption causally imposed a significant and selective effect on metacognitive ability in memory, without altering the perceptual metacognitive performance in the same manner. This suggests the medial parietal cortex might contribute to memory metacognition, in keeping with the established role of the medial parietal cortex in monitoring memory metacognition (Simons et al., 2010; Baird et al., 2013; McCurdy et al., 2013; Morales et al., 2018). However, a recent study identified respective domain-specific and domain-general functional signals engaged by metacognitive judgments in perceptual and memory tasks using multivariate pattern analysis (Morales et al., 2018). They found that the domain-specific pattern for metacognition was encoded in the prefrontal cortex whereas the domaingeneral pattern was distributed in a widespread network in the frontal and posterior midline, including the precuneus. This suggests the precuneus might be dually involved in both memory and perceptual metacognition due to the close relationship shared between the precuneus and perceptual metacognition (McCurdy et al., 2013; Morales et al., 2018). In light of yet another recent report showing that the precuneus contains neural correlates of ERP P3 component during retrospection of task difficulty upon visual perception (Desender et al., 2016), it remains possible that the precuneus is dually involved in the two different domains (McCurdy et al., 2013; Morales et al., 2018) but contributes disproportionally stronger to memory metacognition than otherwise. Desender et al. (2016) interpreted the functional role of the precuneus as to focus attention to the target of metacognition. But given the very limited spatial precision of EEG, and the fact that the metacognitive judgments in their detection paradigm were about the experience of task difficulty, rather than by a 2AFC paradigm tapping into the true introspection of task performance scaled by confidence ratings (Fleming et al., 2010; McCurdy et al., 2013; Morales et al., 2018), a direct comparison with our current findings warrants caution. It is noteworthy that in McCurdy et al. (2013), the most probable model from their data indicates that the precuneus is functionally responsible for only memory but not perceptual metacognition. Here, TMS targeted at the precuneus selectively impairs metacognitive efficiency for memory, but not for perception. Our present study thus causally corroborated the previous model driven by a different method and strengthened domain-specificity of the precuneus in memory metacognition.

Our work carries implications for extending metacognitive principles beyond the realm of working memory to that of episodic memory. The present finding is compatible with other human lesion and neuroimaging studies implicating the role of the parietal cortex in memory retrieval. For example, a lesion study showed that a patient with parietal cortex damage reported feeling less confident and experienced a lack of richness in the memories she retrieved (Davidson et al., 2008). This is consistent with other reports showing that lesions to the parietal cortex significantly diminish retrospective confidence ratings, despite performance remaining intact in a source recollection task (Simons et al., 2010). Furthermore, in a functional neuroimaging study designed to tease apart different components of memory retrieval, activation in the precuneus was found to be associated with vividness judgments during episodic memory retrieval (Richter et al., 2016), consistent with the evidence that the precuneus serves to represent personally relevant content accompanied by vivid recollection (Sreekumar et al., 2017) and detailed abstraction of temporal information required to support recollective TOJ (Ye et al., 2018). Given that vivid reminiscence is a defining feature of 
successful recollection of episodic events, the involvement of the precuneus during memory retrieval tasks might actually lie in its role in subserving the subjective experience of remembering. This argument aligns with the recent finding that EEG activity in the precuneus is linked with conscious dreaming experience (i.e., subjects remembered the content of dreaming experience after being awakened from a dream; Siclari et al., 2017), in line with its role in mental imagery during retrieval (Fletcher et al., 1995). This behavioral and neural evidence convergently implicate the medial parietal cortex in the assessment of recollection during retrieval in support of its role in meta-memory. In line with the contribution to recollection of past episodes, our data corroborated the existing evidence for the participation of the precuneus in higher-order conscious processes during episodic memory retrieval. In a complementary manner, lesions to the anterior PFC are found to impair perceptual metacognitive ability while sparing the metacognitive efficiency for memory, indicating a domain-specific deficit in metacognition by anterior PFC lesions (Fleming et al., 2014). These results conjointly lay the groundwork for a double dissociation in neural areas between memory and perceptual metacognition.

Individual metacognitive efficiency scores were found to be positively correlated across memory and perceptual domains under the control condition in some studies (McCurdy et al., 2013; Ruby et al., 2017; Samaha and Postle, 2017), but not in others (Baird et al., 2013; Vo et al., 2014; Fitzgerald et al., 2017; Sadeghi et al., 2017; Morales et al., 2018). It is plausible that such discord in correlation between metacognitive scores across domains is partly driven by the different types of judgments required (Ruby et al., 2017). A caveat is that the comparison did not take the stimulus characteristics across different tasks into account. Indeed, most studies of metacognition used different categories of materials for the respective tasks, like a word-list memory task versus a dots-contained perceptual task (Baird et al., 2013; McCurdy et al., 2013; Fleming et al., 2014; Sadeghi et al., 2017). Following two recent studies (Ruby et al., 2017; Morales et al., 2018), here we also used stimuli belonging to the same category, and in fact same images, for the memory and perceptual tasks, which would eliminate any confounds attributable to stimulus or featural characteristics.

To conclude, our findings reinforce the notion that precuneal region plays a critical role in mediating metacognition in episodic memory retrieval. To our knowledge, our study is the first one to causally verify the domain-specificity hypothesis of the precuneus in mnemonic metacognition in the human. Together with the contribution of the anterior prefrontal cortex to perceptual metacognition, a challenge for future work is to understand how these different kinds of metacognition can be integrated into a unified framework.

\section{References}

Baird B, Smallwood J, Gorgolewski KJ, Margulies DS (2013) Medial and lateral networks in anterior prefrontal cortex support metacognitive ability for memory and perception. J Neurosci 33:16657-16665. CrossRef Medline

Davidson PS, Anaki D, Ciaramelli E, Cohn M, Kim AS, Murphy KJ, Troyer AK, Moscovitch M, Levine B (2008) Does lateral parietal cortex support episodic memory? Evidence from focal lesion patients. Neuropsychologia 46:1743-1755. CrossRef Medline

Desender K, Van Opstal F, Hughes G, Van den Bussche E (2016) The temporal dynamics of metacognition: dissociating task-related activity from later metacognitive processes. Neuropsychologia 82:54-64. CrossRef Medline

Faivre N, Filevich E, Solovey G, Kühn S, Blanke O (2018) Behavioral, mod- eling, and electrophysiological evidence for supramodality in human metacognition. J Neurosci 38:263-277. CrossRef Medline

Fitzgerald LM, Arvaneh M, Dockree PM (2017) Domain-specific and domain-general processes underlying metacognitive judgments. Conscious Cogn 49:264-277. CrossRef Medline

Flavell JH (1979) Metacognition and cognitive monitoring: a new area of cognitive-developmental inquiry. Am Psychologist 34:906-911. CrossRef

Fleck MS, Daselaar SM, Dobbins IG, Cabeza R (2006) Role of prefrontal and anterior cingulate regions in decision-making processes shared by memory and nonmemory tasks. Cereb Cortex 16:1623-1630. CrossRef Medline

Fleming SM (2017) HMeta-d: hierarchical Bayesian estimation of metacognitive efficiency from confidence ratings. Neurosci Conscious 2017: nix007. CrossRef Medline

Fleming SM, Daw ND (2017) Self-evaluation of decision-making: a general Bayesian framework for metacognitive computation. Psychol Rev 124: 91-114. CrossRef Medline

Fleming SM, Lau HC (2014) How to measure metacognition. Front Hum Neurosci 8:443. CrossRef Medline

Fleming SM, Weil RS, Nagy Z, Dolan RJ, Rees G (2010) Relating introspective accuracy to individual differences in brain structure. Science 329: 1541-1543. CrossRef Medline

Fleming SM, Ryu J, Golfinos JG, Blackmon KE (2014) Domain-specific impairment in metacognitive accuracy following anterior prefrontal lesions. Brain 137:2811-2822. CrossRef Medline

Fletcher PC, Frith CD, Baker SC, Shallice T, Frackowiak RS, Dolan RJ (1995) The mind's eye: precuneus activation in memory-related imagery. Neuroimage 2:195-200. CrossRef Medline

Frith CD (2012) The role of metacognition in human social interactions. Philos Trans R Soc Lond B Biol Sci 367:2213-2223. CrossRef Medline

Galvin SJ, Podd JV, Drga V, Whitmore J (2003) Type 2 tasks in the theory of signal detectability: discrimination between correct and incorrect decisions. Psychon Bull Rev 10:843-876. CrossRef Medline

Green DM, Swets JA (1966) Signal detection theory and psychophysics. New York: John Wiley \& Sons.

Iyer MB, Schleper N, Wassermann EM (2003) Priming stimulation enhances the depressant effect of low-frequency repetitive transcranial magnetic stimulation. J Neurosci 23:10867-10872. CrossRef Medline

Kornell N, Son LK, Terrace HS (2007) Transfer of metacognitive skills and hint seeking in monkeys. Psychol Sci 18:64-71. CrossRef Medline

Kraft A, Dyrholm M, Kehrer S, Kaufmann C, Bruening J, Kathmann N, Bundesen C, Irlbacher K, Brandt SA (2015) TMS over the right precuneus reduces the bilateral field advantage in visual short term memory capacity. Brain Stimul 8:216-223. CrossRef Medline

Kwok SC, Shallice T, Macaluso E (2012) Functional anatomy of temporal organisation and domain-specificity of episodic memory retrieval. Neuropsychologia 50:2943-2955. CrossRef Medline

Maniscalco B, Lau H (2012) A signal detection theoretic approach for estimating metacognitive sensitivity from confidence ratings. Conscious Cogn 21:422-430. CrossRef Medline

McCurdy LY, Maniscalco B, Metcalfe J, Liu KY, de Lange FP, Lau H (2013) Anatomical coupling between distinct metacognitive systems for memory and visual perception. J Neurosci 33:1897-1906. CrossRef Medline

Morales J, Lau H, Fleming SM (2018) Domain-general and domain-specific patterns of activity supporting metacognition in human prefrontal cortex. J Neurosci 38:3534-3546. CrossRef Medline

Nelson TO (1990) Metamemory: a theoretical framework and new findings. In: Psychology of learning and motivation (Bower GH, ed), pp 125-173. New York: Academic.

Nilakantan AS, Bridge DJ, Gagnon EP, VanHaerents SA, Voss JL (2017) Stimulation of the posterior cortical-hippocampal network enhances precision of memory recollection. Curr Biol 27:465-470. CrossRef Medline

Rahnev D, Nee DE, Riddle J, Larson AS, D’Esposito M (2016) Causal evidence for frontal cortex organization for perceptual decision making. Proc Natl Acad Sci U S A 113:6059-6064. CrossRef Medline

Richter FR, Cooper RA, Bays PM, Simons JS (2016) Distinct neural mechanisms underlie the success, precision, and vividness of episodic memory. eLife 5:e18260. CrossRef Medline

Rossi S, Hallett M, Rossini PM, Pascual-Leone A (2009) Safety, ethical considerations, and application guidelines for the use of transcranial magnetic stimulation in clinical practice and research. Clin Neurophysiol 120:2008-2039. CrossRef Medline 
Rossini PM, Burke D, Chen R, Cohen LG, Daskalakis Z, Di Iorio R, Di Lazzaro V, Ferreri F, Fitzgerald PB, George MS, Hallett M, Lefaucheur JP, Langguth B, Matsumoto H, Miniussi C, Nitsche MA, Pascual-Leone A, Paulus W, Rossi S, Rothwell JC, et al. (2015) Non-invasive electrical and magnetic stimulation of the brain, spinal cord, roots and peripheral nerves: basic principles and procedures for routine clinical and research application: an updated report from an I.F.C.N. Committee. Clin Neurophysiol 126:1071-1107. CrossRef Medline

Rounis E, Maniscalco B, Rothwell JC, Passingham RE, Lau H (2010) Thetaburst transcranial magnetic stimulation to the prefrontal cortex impairs metacognitive visual awareness. Cogn Neurosci 1:165-175. CrossRef Medline

Ruby E, Giles N, Lau H (2017) Finding domain-general metacognitive mechanisms requires using appropriate tasks. bioRxiv 211805. CrossRef

Sadeghi S, Ekhtiari H, Bahrami B, Ahmadabadi MN (2017) Metacognitive deficiency in a perceptual but not a memory task in methadone maintenance patients. Sci Rep 7:7052. CrossRef Medline

Samaha J, Postle BR (2017) Correlated individual differences suggest a common mechanism underlying metacognition in visual perception and visual short-term memory. Proc Biol Sci 284:20172035. CrossRef Medline

Siclari F, Baird B, Perogamvros L, Bernardi G, LaRocque JJ, Riedner B, Boly M, Postle BR, Tononi G (2017) The neural correlates of dreaming. Nat Neurosci 20:872-878. CrossRef Medline

Simons JS, Peers PV, Mazuz YS, Berryhill ME, Olson IR (2010) Dissociation between memory accuracy and memory confidence following bilateral parietal lesions. Cereb Cortex 20:479-485. CrossRef Medline
Sreekumar V, Nielson DM, Smith TA, Dennis SJ, Sederberg PB (2017) The experience of vivid autobiographical reminiscence is supported by personal semantic representations in the precuneus. bioRxiv 197665. CrossRef

Teasdale JD, Moore RG, Hayhurst H, Pope M, Williams S, Segal ZV (2002) Metacognitive awareness and prevention of relapse in depression: empirical evidence. J Consult Clin Psychol 70:275-287. CrossRef Medline

Thut G, Pascual-Leone A (2010) A review of combined TMS-EEG studies to characterize lasting effects of repetitive TMS and assess their usefulness in cognitive and clinical neuroscience. Brain Topogr 22:219-232. CrossRef Medline

Vo VA, Li R, Kornell N, Pouget A, Cantlon JF (2014) Young children bet on their numerical skills: metacognition in the numerical domain. Psychol Sci 25:1712-1721. CrossRef Medline

Wang JX, Rogers LM, Gross EZ, Ryals AJ, Dokucu ME, Brandstatt KL, Hermiller MS, Voss JL (2014) Targeted enhancement of cortical-hippocampal brain networks and associative memory. Science 345:1054-1057. CrossRef Medline

Ye Q, Hu Y, Ku Y, Appiah K, Kwok SC (2018) Locally distributed abstraction of temporal distance in human parietal cortex. bioRxiv 249904. CrossRef

Yokoyama O, Miura N, Watanabe J, Takemoto A, Uchida S, Sugiura M, Horie K, Sato S, Kawashima R, Nakamura K (2010) Right frontopolar cortex activity correlates with reliability of retrospective rating of confidence in short-term recognition memory performance. Neurosci Res 68:199-206. CrossRef Medline 